\title{
EXPLORER LES POSSIBLES DE L'ÉCRITURE MULTIMÉDIA
}

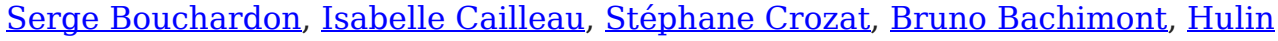 \\ Thibaud
}

GRESEC | «Les Enjeux de l'information et de la communication »

$2011 / 2 n^{\circ} 12 / 2 \mid$ pages 11 à 24

\section{Article disponible en ligne à l'adresse :}

https://www.cairn.info/revue-les-enjeux-de-l-information-et-de-lacommunication-2011-2-page-11.htm

Distribution électronique Cairn.info pour GRESEC.

(C) GRESEC. Tous droits réservés pour tous pays.

La reproduction ou représentation de cet article, notamment par photocopie, n'est autorisée que dans les limites des conditions générales d'utilisation du site ou, le cas échéant, des conditions générales de la licence souscrite par votre établissement. Toute autre reproduction ou représentation, en tout ou partie, sous quelque forme et de quelque manière que ce soit, est interdite sauf accord préalable et écrit de l'éditeur, en dehors des cas prévus par la législation en vigueur en France. Il est précisé que son stockage dans une base de données est également interdit. 


\title{
Explorer les possibles de l'écriture multimédia
}

Article inédit. Mis en ligne le 16 décembre 2011.

\author{
Auteurs : Serge Bouchardon*, Isabelle Cailleau*, Stéphane Crozat**, Bruno \\ Bachimont***, Thibaud Hulin* \\ Université de Technologie de Compiègne \\ * EA 2223 COSTECH \\ ** Unité Ingénierie des Contenus et Savoirs \\ *** UMR CNRS 6599 Heudiasyc \\ Agrégé de lettres modernes, chef de projet pendant six ans dans l'industrie du multimédia éducatif, Serge \\ Bouchardon est actuellement maître de conférences en sciences de l'information et de la communication à \\ l'Université de Technologie de Compiègne. \\ Parmi ses champs de recherche : l'écriture interactive et multimédia, la création numérique. \\ Dernier ouvrage paru : Littérature numérique : le récit interactif, Hermès Lavoisier, Paris, décembre 2009.
}

Plan

Une approche conceptuelle de l'écriture multimédia

Etudes de cas

Conclusion

Références bibliographiques

\section{Résumé}

Dans cet article, nous posons la question d'une écriture multimédia et de ses spécificités. Le multimédia permet-il d'ouvrir des possibles pour l'écriture ? Pour comprendre et explorer ces possibles, nous mobilisons une théorie du numérique et proposons un modèle en trois niveaux de l'écriture multimédia. Nous utilisons cette approche conceptuelle pour analyser des pratiques éditoriales professionnelles à l'Institut national de l'audiovisuel (Ina).

Mots-clés : écriture multimédia, numérique, approche conceptuelle, Institut national de l'audiovisuel, INA

\section{Abstract}

In this article, we shall pose the question of multimedia writing and of its specificities. Does multimedia open a new realm of possibilities for writing? Our approach is based on a theory of the Digital which offers a three level model of multimedia writing. We shall use this conceptual approach to analyse the professional editorial practices at the National Audiovisual Institute.

Keywords: multimedia writing, digital, conceptual approach, the National Audiovisual Institute, INA. 
Dans nos discours ordinaires, le terme multimédia tend à devenir le simple synonyme de numérique. Il renvoie pourtant plus précisément à la manipulation conjointe de textes, images et sons sur un même support. Se pose alors la question d'une écriture multimédia et de ses spécificités. Le multimédia permet-il d'ouvrir des possibles pour l'écriture, ces « moyens que l'homme a trouvés pour rendre sa langue visible » (Christin, 2001) et plus que jamais manipulable ? Pour comprendre et explorer ces possibles, nous mobiliserons une théorie du numérique et proposerons un modèle en trois niveaux de l'écriture multimédia. Nous utiliserons cette approche conceptuelle pour analyser des pratiques éditoriales professionnelles à l'Institut national de l'audiovisuel (Ina).

\section{UNE APPROCHE CONCEPTUELLE DE L'ECRITURE MULTIMEDIA}

La question de l'écriture s'articule à celle des contenus, c'est-à-dire d'objets matériels reposant sur un support physique de manifestation pour donner à interpréter une forme considérée comme signifiante, forme que l'on peut dès lors appeler "forme sémiotique » (Bachimont, 2007). L'écriture est dans cette perspective un système technique permettant la création, la manipulation et l'exploitation de contenus (la lecture ne pouvant être dissociée de l'écriture). Dans l'écriture se noue une tension essentielle entre le support physique de manifestation et la forme sémiotique d'interprétation: les conditions matérielles de la première conditionnent l'intelligibilité de la seconde dans la mesure où d'une part elle configure les formes qui seront données à percevoir et où d'autre part elle conditionne les procédures d'exploitation, d'interaction, et d'utilisation de ses formes. D'un volumen que l'on déroule à un codex dont on tourne les pages, jusqu'au texte numérique que l'on fait défiler, les conditions phénoménologiques de la lecture et de l'écriture sont radicalement modifiées.

Il appert que l'analyse de l'écriture, d'un type d'écriture, doit en passer par l'analyse des propriétés de son support d'inscription, même si elle ne peut s'y réduire. Cette analyse est par nature difficile : outre les dénis classiques mais idéologiques du rôle de la technique et de ses supports dans la genèse phénoménologique de la cognition et de la pensée, dénis qu'il faut imputer à une peur mêlée d'incompréhension des fondamentaux technoscientifiques qui structurent notre environnement, le procès de l'écriture repose par nature sur un substrat technique qui est difficile à isoler du fait de son évidence et de sa présence. L'occasion d'observer le rôle du support se manifeste lors d'un changement technique qui altère suffisamment ses propriétés matérielles pour que les conséquences cognitives se manifestent via leur rupture vis-à-vis des pratiques précédentes.

Goody (Goody, 1979) a ainsi montré que la culture de l'écrit a transformé nos capacités cognitives. En proposant un support de manifestation spatial et non temporel, synoptique et non séquentiel, l'écrit modifie les modes d'appréhension phénoménologique du contenu et permet la constitution de catégories cognitives inédites (notamment la liste, la formule, le tableau), ouvrant le principe d'une pensée systématique de la classification. Le numérique opère sur les mêmes modalités en élargissant la synopsis spatiale, rendant accessible par le calcul ce qui est séparé selon le devenir, stockant et rendant disponible les formes sémiotiques éphémères parce que temporelles (son, audiovisuel, etc., à la suite des premières techniques analogiques d'enregistrement). Dans cette perspective, le numérique devrait entraîner une transformation de nos modes de pensée. C'est l'hypothèse défendue par Bruno Bachimont : "Si l'écriture a donné lieu à une raison graphique, le numérique doit donner lieu à une raison computationnelle: le calcul comme technique de manipulation de symboles entraîne un mode spécifique de pensée, qui ne remplace pas les 
autres, mais les reconfigure »(Bachimont, 2000, p.3) ${ }^{1}$. Notre démarche est ainsi la conséquence d'un positionnement théorique qui pose que les pratiques se construisent en fonction de possibles techniques confrontés à des usages, et donc que le support matériel joue un rôle dans la structuration des connaissances.

La question principale est donc de comprendre ce que fait le numérique aux contenus dès lors que l'on recourt à ce type de support. Dans le cadre du projet PRECIP (http://precip.fr) financé par la Région Picardie, nous proposons une analyse du numérique à finalité heuristique pour caractériser cette influence, analyse heuristique qui nous sert à décliner un modèle de l'écriture multimédia. Cette analyse repose sur une distinction de 3 niveaux :

- niveau théorético-idéal : c'est le numérique comme principe calculatoire qui a pour tendance de réduire les contenus à une combinatoire aveugle de signes privés de sens, les 0 ou les 1 , ou n'importe quelles unités logiques formelles constituant un alphabet de manipulation.

Mais dès qu'on utilise le numérique, ce n'est pas seulement pour profiter de ses propriétés calculatoires, c'est aussi pour les appliquer sur des entités possédant une réalité matérielle et un sens interprétatif. L'enjeu est alors de savoir sortir de l'autarcie du numérique pour le renégocier dans le monde de la matière et du sens. D'une part, on le matérialise, c'est l'implémentation, d'autre part on l'utilise pour manipuler des contenus et interagir avec le système. On distingue alors deux niveaux selon que l'on s'intéresse au support matériel de manifestation et à sa forme sémiotique d'interprétation, ou bien aux formats d'interaction et à ses formes d'usage associées.

On distingue alors :

- un niveau techno-applicatif, niveau de la manifestation : il s'agit de mobiliser le numérique sur des contenus via des formats; les formats correspondent à la structuration sous forme calculable ou manipulable du contenu: les alphabets comme Unicode ou ASCII, les codages comme JPEG ou MPEG, etc. Ce niveau introduit une tension entre la forme d'expression du contenu et la structuration que les formats lui imposent.

- Un niveau sémio-rhétorique, niveau de l'interaction : le contenu numérisé s'accompagne d'une manière de faire sens héritée de son instrumentation numérique liée à l'interaction proposée, les manipulations rendues possibles, les parcours interprétatifs induits. On constate une tension entre les stéréotypes du discours et de la construction de sens et les écarts induits tant par l'écriture que par l'interprétation, dans la mesure où ce qui fait sens se construit par écart à la norme, cette norme étant le préalable conventionnel par rapport auquel se situer pour s'en démarquer.

On voit alors que le numérique comme tel, objet idéal comme calculabilité et combinatoire, prend une existence matérielle via l'implémentation et une consistance

\footnotetext{
${ }^{1}$ Il ne s'agit pas ici d'un déterminisme technique. Nous reprenons en effet la thèse de Bruno Bachimont pour qui, « de manière générale, la structure physique et matérielle de l'inscription conditionne son interprétation, mais ne la programme ni ne la détermine à l'avance » (Bachimont, 2004, p.119).
} 
pratique via la manifestation d'un contenu sous une forme sémiotique perceptible et via l'interaction qu'il permet.

\section{Le niveau théorético-idéal}

Le premier niveau est celui du numérique théorique : il définit les possibles du support numérique d'un point de vue théorique. Contrairement aux deux autres niveaux, il est purement idéal. En effet, le numérique - en tant que calculabilité définie comme manipulation formelle - est une abstraction logique (Bachimont, 2007) qui n'existe pas concrètement : en pratique, on a toujours affaire à des symboles matériels physiquement réalisés (implémentation) et perceptiblement montrés (interfaces de manifestation et d'interaction).

La manipulation porte sur des unités discrètes qui sont codées de manière arbitraire en 0 et 1 (code binaire) et sont dépourvues de sémantique a priori. Elles vont ainsi pouvoir être manipulées selon des règles formelles indépendamment de toute sémantique, si bien que ce premier niveau se caractérise par deux principes fondamentaux : la discrétisation et la manipulation.

Le niveau théorético-idéal correspond au fait que tout contenu, dès lors qu'il est numérique ou numérisé, peut être réduit à un code calculable, manipulable, dont la signification éventuelle est arbitraire et indépendante de la manipulation formelle. Dans cette optique, le contenu devient un code anonyme manipulable de manière aveugle, de manière totalement arbitraire par rapport à la signification que l'on peut associer au contenu. Si bien que le caractère numérique introduit une tension entre l'ouverture d'un horizon de manipulations arbitraires possible et le fait que seules certaines manipulations seront signifiantes.

\section{Le niveau techno-applicatif}

Ce niveau se caractérise par un formatage du binaire (c'est-à-dire de l'alphabet formel) pour construire de manière a priori un espace des possibles dans un registre donné de contenus. Par exemple, le terme unicode désigne un format qui prescrit a priori l'expression alphabétique en numérique, le MPEG désigne un format pour la vidéo, etc. À ce stade, le format spécialise et concrétise l'idéalité du numérique en un espace de manipulation possible défini sur des unités élémentaires fixées par le format : le caractère pour Unicode, le pixel en format TIFF, le GOP (Group of Picture) en MPEG, etc. Ces unités sont déterminées a priori et le format définit ce qu'il est possible de faire avec elles. En ce sens, on a bien un niveau techno-applicatif et non interprétatif : nous n'avons pas un parcours interprétatif définissant a posteriori les signes et leur signifiance et leur sens, mais un formatage a priori qui prescrit des unités et leur manipulation formelle.

Il s'instaure alors une tension entre le format, technique et mobilisant des unités a priori, et les formes sémiotiques manifestées par ces formats, formes qui sont interprétatives et dégageant a posteriori les unités de sens. Ce qui est manipulable n'est pas directement ce qui est signifiant, ce qui est signifiant n'est pas directement ce qui est manipulable. Quand c'est le cas, c'est qu'on l'a forcé ou contraint via un format logique qui force la coïncidence entre le format technique et la forme sémiotique, la sémantique étant pilotée par la syntaxe; cas exceptionnel que l'on rencontre dans les bases de données, dans les formalismes du Web sémantique, mais pas dans les contenus usuels (textes, images, sons, etc.). 


\section{Le niveau sémio-rhétorique}

Au-delà du contenu manifesté via des formats de codage et de manipulation, le numérique permet d'interagir avec le contenu et les fonctions du système qui le contiennent. À ce stage, on trouve des schémas d'interaction, des fonctionnalités proposées à l'interaction par l'utilisateur qui structurent sa pratique. Ces schémas se traduisent par les scripts, les workflows, les tâches, que l'on trouve dans les systèmes et qui " programment » l'usage. De la même manière que l'on a une combinatoire induite par le codage, on a une combinatoire des fonctions d'interaction.

Ces fonctions et leur combinatoire entrent en tension avec les pratiques effectives et l'usage qui, par nature, détourne et altère les fonctions prévues des effets de co

ntexte et de l'interprétation qu'il conditionne. L'interaction effective dégage du sens qui n'est jamais le choix d'une modalité parmi les multiples possibilités prévues à l'avance, mais une innovation qui sort de l'horizon des possibles ouvert par la combinatoire.

Ces trois niveaux entrent en tension entre eux, et possèdent par eux-mêmes leur propre tension. Le niveau théorético-idéal connaît la tension entre le format formel idéal et le substrat physique sous-jacent, c'est la tension de l'implémentation. Le niveau technoapplicatif connaît la tension entre le format de codage et la forme d'interprétation, c'est la tension de la manifestation. Le niveau sémio-rhétorique connaît la tension entre les fonctions du système et la pratique d'usage, c'est la tension de l'interaction.

Par exemple, au niveau techno-applicatif, la forme sémiotique vidéo présente des contraintes de lecture : en effet, une séquence vidéo fait sens lorsqu'elle est regardée du début à la fin. Elle propose une globalité linéaire, avec une certaine progression. Or, du point de vue du format, il est possible de proposer une fragmentation, inhérente à la logique de manipulation. Lorsque cette fragmentation est appliquée à la vidéo se pose la question du sens : comment reconstruire du sens à partir de cette fragmentation ? Que devient la forme sémiotique dès lors qu'elle est formatée et fragmentée ?

De même, au niveau sémio-rhétorique, les fonctions d'interaction auront tendance à séparer les fonctions d'écriture des fonctions de mises en forme, séparant d'une certaine manière le fond et la forme. Pourtant, la pratique de l'écriture révèle que la manière d'écrire influe sur la nature de ce que l'on écrit, les soucis de mise en forme faisant partie de l'expression du contenu. Ce sont des difficultés que l'on retrouvera dans les systèmes documentaires et les chaînes éditoriales où les auteurs se voient proposer une logique rationnelle du point de l'outil mais contre-intuitive du point de vue de l'écriture. C'est la raison pour laquelle un outil comme LaTeX reste si difficile à manipuler pour le néophyte, séparant en deux étapes distinctes (la rédaction et la mise en forme) des tâches fortement intégrées. Malgré tous ses défauts, un traitement de texte comme Word® reste plus accessible de ce point de vue.

Enfin, entre les niveaux, on trouvera une tension entre le techno-applicatif et le sémiorhétorique: d'un point de vue technique, il faut que les fonctions d'interaction soient constructibles à partir des formats de codage. De même, la pratique d'usage qui en découle doit pouvoir s'articuler avec les formes sémiotiques de manifestation. En reprenant l'exemple de la vidéo, la fragmentation permet de donner lieu à de nouvelles formes sémiotiques, que l'on voit fleurir sur le Web par exemple, et qui induisent des pratiques inédites. Les tensions sont ainsi productives et reconfigurent tant les contenus que leur signifiance. On peut voir ainsi émerger le film interactif, dans lequel la vidéo serait délinéarisée voire documentée à l'aide d'autres ressources. 
Si ces 3 niveaux sont autonomes, ce sont les rapports et les tensions entre ces trois niveaux qui font émerger des formes d'écriture multimédia. Cette analyse se décline en un modèle de l'écriture multimédia, modèle qui est ainsi avant tout le nom d'un problème. Étant donnés les possibles théoriques et les potentiels applicatifs du numérique, comment des médias différents (texte, image, son, vidéo) vont-ils faire signe et sens ensemble dans un contexte de communication donné ?

Au niveau techno-applicatif notamment, les formats sont exploités pour la programmation de fonctions d'écriture. Cela se concrétise en propriétés techniques (manipulabilité, abstraction, adressabilité, universalité, clonabilité) déclinées en fonctions² ${ }^{2}$ Par exemple, la propriété de la manipulabilité propose une interactivité, qui en termes de fonctions d'écriture peut prendre la forme d'une programmation de parcours multiples (l'utilisateur a par exemple le choix entre plusieurs niveaux de lecture, tels un mode débutant ou un mode expert), ou encore la forme d'une introduction de données (l'utilisateur est invité à entrer des données, par exemple la réponse à une question, et le contenu est reconfiguré en fonction de la réponse). Ces propriétés sont issues d'une approche inductive, d'une observation et d'une généralisation à partir des fonctions implémentées dans les applications actuelles. Ce potentiel applicatif a donc une forte dimension socio-historique : il est en constante évolution.

Ces niveaux d'analyse de l'écriture multimédia permettent de mieux comprendre les objets numériques et en même temps de caractériser l'écriture multimédia en observant ces contenus dans l'optique de cette grille d'analyse. C'est ce à quoi nous allons nous livrer à présent.

\section{ÉTUDES DE CAS}

Dans cette seconde partie, nous mobilisons notre approche conceptuelle afin d'éclairer $a$ posteriori les pratiques réelles de professionnels. Nous illustrons ainsi la pertinence de notre approche en trois niveaux pour comprendre les ressorts de deux situations d'écriture multimédia, à l'Institut national de l'audiovisuel (Ina), avec la chaîne éditoriale Scenari (scenari-platform.org). Le premier cas porte sur les Fresques Hypermédias (www.ina.fr/dossier/fresques) : nous verrons comment s'articule le travail des informaticiens avec celui des historiens pour valoriser un fonds documentaire. Le second cas porte sur la Webradio du Groupe de Recherches Musicales (GRM), qui valorise des enregistrements sonores en leur redonnant une nouvelle vie (www.inagrm.com).

Ces cas ont pu être étudiés dans le cadre du projet ANR C2M - auquel participent l'UTC et l'Ina - dont l'objectif est l'étude et la conception de systèmes d'écriture multimédia collaboratifs (scenari.utc.fr $/ \mathrm{c}^{2} \mathrm{~m}^{3}$ ). Une expression de pratiques et de besoins a été formulée par les acteurs de l'Ina (Saint-Martin \& al., 2011) et des entretiens spécifiques ont été effectués pour l'analyse plus ciblée par cet article.

\footnotetext{
${ }^{2}$ Une carte du niveau techno-applicatif est disponible ici : http://precip.fr/map

3 Voir en particulier http://scenari.utc.fr/c2m/co/grm.html et http://scenari.utc.fr/c2m/co/studio.html pour les pilotes réalisés avec l'Ina.
} 


\section{Fresques : conception d'un modèle applicatif pour l'écriture multimédia}

\section{Mise en contexte}

L'Ina possède un fonds de documents audiovisuels numérisés qu'il peut mettre à disposition dans le cadre de partenariats. Une de ces exploitations se fait sous la forme de Fresques hypermédias (www.ina.fr/dossier/fresques). La réutilisation d'archives dans ce contexte suppose une "ré-éditorialisation» (c'est-à-dire une réécriture qui permet de s'adapter au nouveau contexte de publication) qui dépasse la simple juxtaposition de ressources (Gaillard \& Crozat, 2011, p. 65). Des opérations d'écriture sont convoquées, telles que : «l'adjonction de métadonnées », « la juxtaposition avec d'autres documents » ou « l'ajout d'introduction, de transitions, de conclusion» (op. cit.).

Sur le plan technique, l'Ina utilise la chaîne éditoriale XML Scenari (Crozat, 2007), un logiciel libre destiné à la composition de documents multimédias, se fondant sur leur structuration logique plutôt que leur présentation physique. Ainsi, une description logique d'un contenu permet ensuite d'en produire diverses présentations (animation interactive pour le Web, diaporama pour une diffusion en présence de spectateurs, notice statique imprimable...). Pour cela, un «modèle » d'écriture qui va structurer l'écrit multimédia a été préalablement créé, qui détermine les schèmes de représentation du contenu. Ce modèle est exprimé selon un formalisme informatique (on parle de schéma ou grammaire documentaire, voir par exemple www.w3.org/XML/Schema) qui encadre la production de fichiers XML conformes (dit «valides ») à ce qui attendu en terme de structure.

Un modèle répond à un objectif éditorial spécifique : ainsi le modèle des Fresques est destiné à promouvoir et à remettre en scène un fonds documentaire audiovisuel. Il est donc nécessaire, lors de la conception de ce modèle, d'établir les règles précises qui encadrent la pratique d'écriture visée : quels documents peuvent être sélectionnés, comment les décrire à l'aide de méta-données, comment rédiger les notices et les enrichir d'une documentation complémentaire... Il faut également concevoir les publications (mises en forme finales), en l'occurrence penser la manière dont la présentation d'un ensemble de fragments multimédias fera sens pour le public visé.

Pour produire ses Fresques, l'Ina met en place une équipe pluridisciplinaire orientée vers la valorisation du fonds : les informaticiens développent le modèle (schéma de structuration et formats de présentation) ; les rédacteurs, par exemple des universitaires spécialistes du contenu, rédigent un guide de recherche dans un premier temps, puis des mises en contexte et des parcours thématiques, une fois les vidéos sélectionnées ; les documentalistes sélectionnent des archives à partir du guide de recherche, puis, une fois celles-ci validées, les documentarisent (ou redocumentarisent), en particulier par la mise à jour de métadonnées ; enfin un comité éditorial est chargé de coordonner le projet et d'en assurer la cohésion globale (validation du guide de recherche, des archives sélectionnées, des notices rédigées et des parcours). 
Figure 1. Une Fresque hypermédia de l'Ina

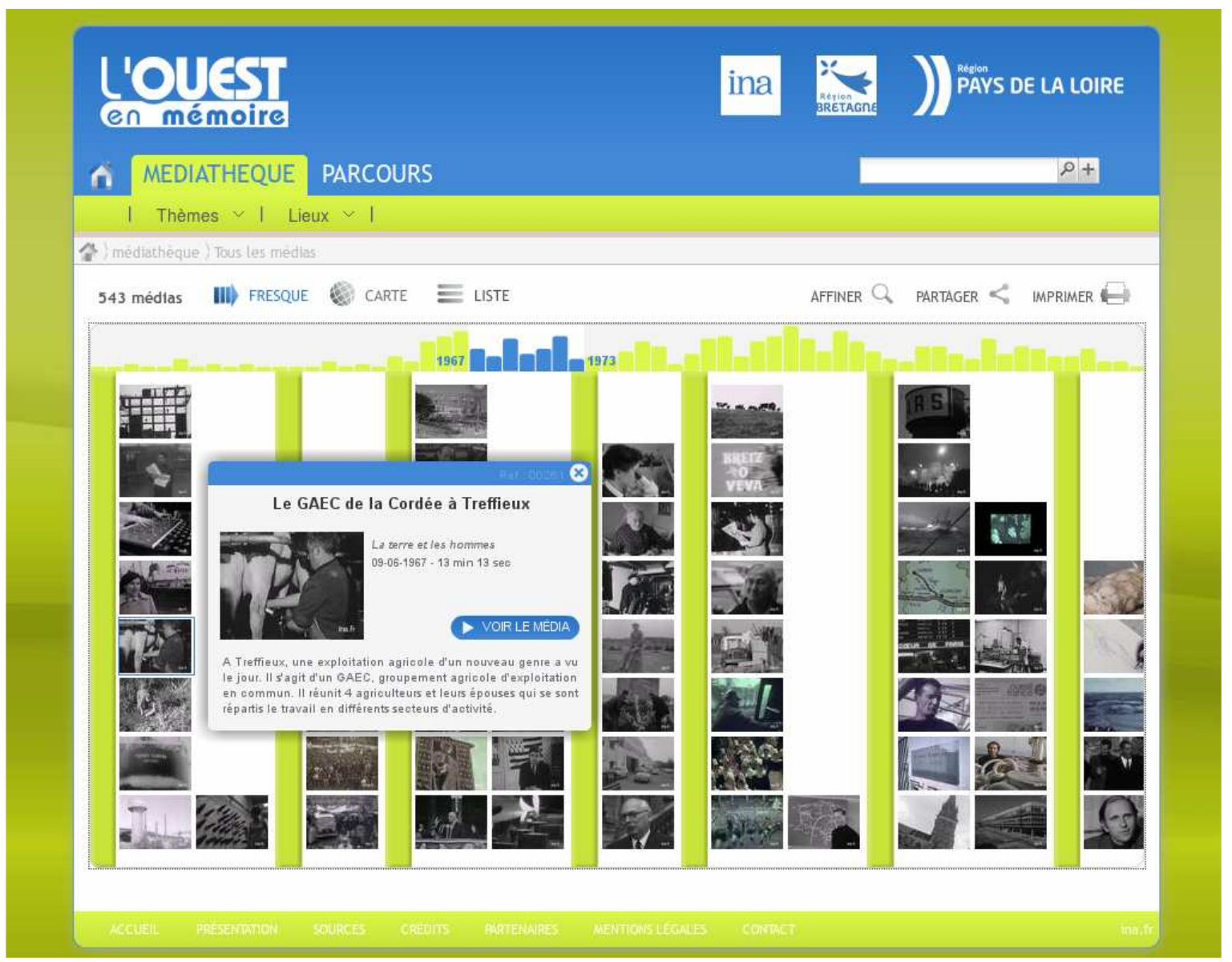

\section{Éléments d'analyse}

La qualité avérée des Fresques, repose sur - outre l'expertise des équipes - la manière dont les tensions sont gérées entre les trois niveaux du numérique. En particulier sur la manière dont les possibles sont intériorisés par les acteurs, et dont ils sont négociés par rapport au contexte.

Le travail d'écriture des Fresques se découpe en plusieurs moments distincts : la conception du modèle, la sélection des archives, la redocumentarisation des archives, et la rédaction de documents complémentaires pour l'indexation et de parcours thématiques.

Premièrement, le principe même du modèle de document s'appuie sur des propriétés propres au numérique : le document est pensé comme une articulation d'unités distinctes, discrètes et manipulables (les parties, les ressources audiovisuelles...). On peut alors élaborer un ensemble de règles formelles a priori qui vont structurer l'ensemble des documents à rédiger. Le respect de ces règles va pouvoir être contrôlable par calcul. On pourra également établir des algorithmes de transformation pour présenter les contenus selon les présentations finales recherchées, par exemple la structure arborescente des contenus va être transformée dans la forme tabulaire de la fresque (cf. figure 1). L'approche par modèle montre une adéquation forte avec le numérique qui permet donc son contrôle et la manipulation automatique des instances. 
Deuxièmement, la sélection des vidéos est guidée par le sujet du projet de rééditorialisation. Les rédacteurs rédigent un guide de recherche indépendamment du fonds, puis les documentalistes effectuent une sélection de 400 à 500 documents. Là encore, c'est une fonction fondamentale du numérique, l'abstraction, qui est mobilisée. Elle permet de manipuler une représentation des vidéos (titre, résumé, durée, transcription...) comme si c'était l'objet lui-même. Ainsi, le travail documentaire peut se faire sur des abstractions plus simples à manipuler que les objets eux même, a fortiori lorsque l'on parle de vidéos (des URL dans une base de données par exemple).

Troisièmement, les documentalistes entament un travail de rédaction de documents secondaires et d'indexation fondés sur des thésaurus. Afin d'appréhender de nombreuses vidéos, issues d'un fonds très large, il est en effet nécessaire de les décrire par des énoncés textuels (les notices) plus facilement manipulables par la machine et lisibles synthétiquement par l'homme. On produit des descripteurs comme des dates, des résumés, des transcriptions, le type de document concerné, l'état de la diffusion, l'auteur, la langue, etc. Des propriétés fondamentales du numérique sont à nouveau mobilisées, comme la possibilité d'intégrer sur un même support devenu universel grâce au codage binaire, le contenu audiovisuel et ses métadonnées descriptives ; la possibilité de calculer formellement sur les métadonnées (par exemple pour les apparier avec l'énoncé d'une requête) ; ou encore la possibilité d'adresser des moments particuliers de la vidéo (timecode).

Quatrièmement, les rédacteurs vont constituer des parcours dans l'ensemble hétérogène de documents. Un parcours est une réponse éditoriale à une intention de communication. Tandis que la Fresque invite plutôt au feuilletage du contenu, le parcours prescrit un ordre de lecture et propose une mise en contexte (cf. figure 2). Ici encore les propriétés du numérique sont exploitées : l'adressabilité numérique permet de compulser un ensemble de fragments audiovisuels, en activant la lecture à partir d'une image ou d'un descripteur de la vidéo ; la même adressabilité dote la ressource d'ubiquité et lui permet de se trouver à plusieurs endroits en même temps. 
Figure 2. Proposition d'un "parcours" dans une Fresque

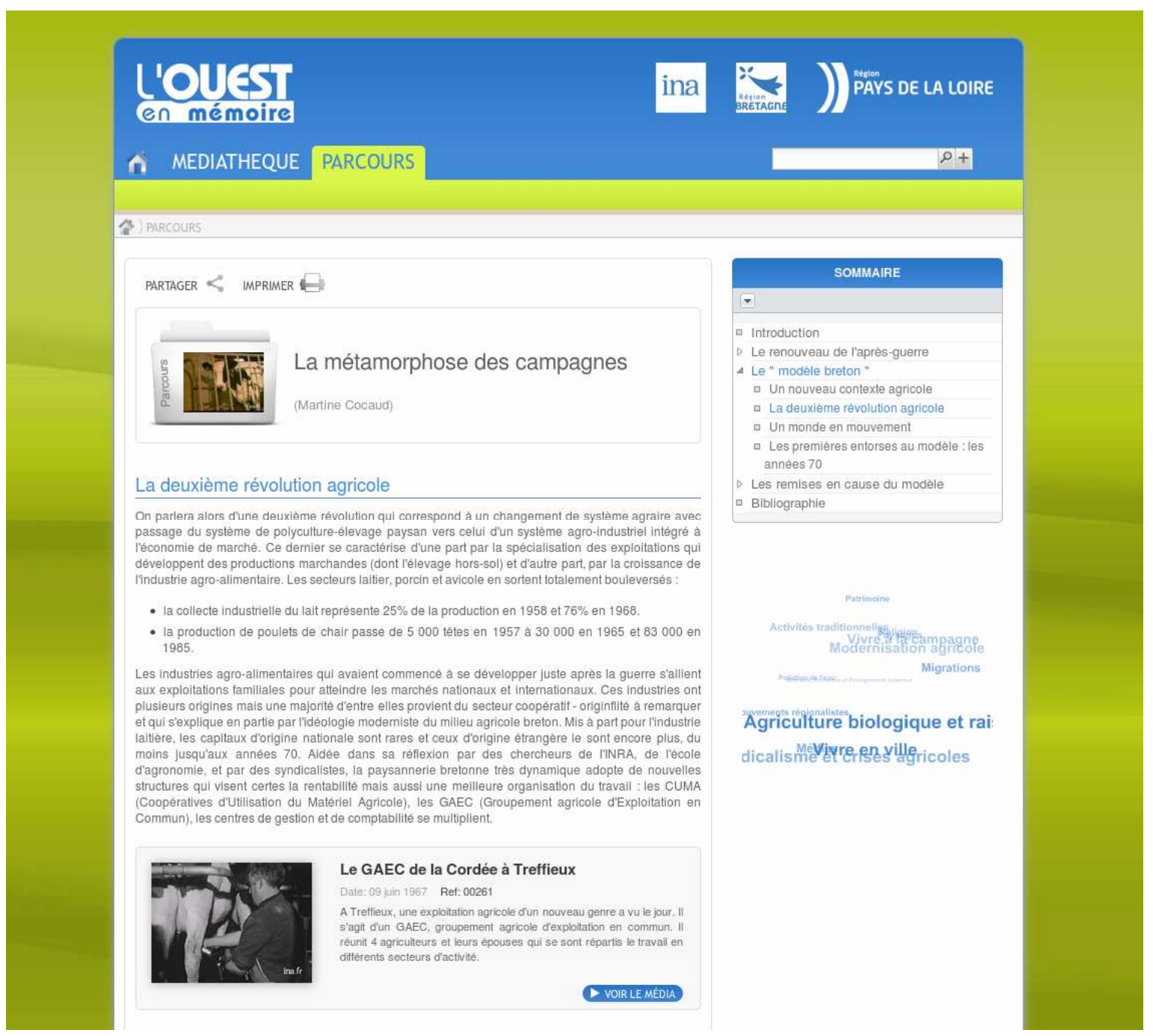

Cette première analyse montre comment les choix effectués par les professionnels résultent d'une mobilisation pertinente des trois niveaux et d'une gestion des tensions entre ceux-ci.

\section{Webradios : enrichissement contraint d'un flux sonore}

\section{Mise en contexte}

Le Groupe de Recherches Musicales (GRM) de l'Ina est à l'origine d'un autre dispositif de valorisation d'enregistrements, ceux de la radio. Le GRM est pionnier de la musique électroacoustique. Il valorise des archives sur ce thème et produit des écritures originales, sous la forme de Webradios (http://www.inagrm.com/grm-webradios), émissions de radio ré-éditorialisées pour le Web, navigables et enrichies, avec le même outil Scenari, mais selon un autre modèle (éponyme, cf. . http://scenari-platform.org/projects/webradio/fr/pres).

Les Webradios du GRM présentent des documents multimédias interactifs qui articulent des enregistrements audio avec des photographies et des documents complémentaires. Par rapport aux Fresques, les Webradios favorisent la consultation de documents en même temps que l'on écoute la bande sonore. En effet, il s'agit ici d'enrichir un flux sonore par l'ajout de documents tiers : photographies, textes, liens hypertextes... voire de nouveaux flux 
audio ou vidéo complémentaires (qui interrompent alors l'écoute du flux maître). Les Webradios proposent également une discrétisation de l'écoute, à travers un plan de l'émission (chapitrage) et la possibilité de naviguer dans le flux via celui-ci (liens à des timecodes donnés).

Figure 3. Une émission Webradio : segmentation et enrichissement multimédia du flux audio.

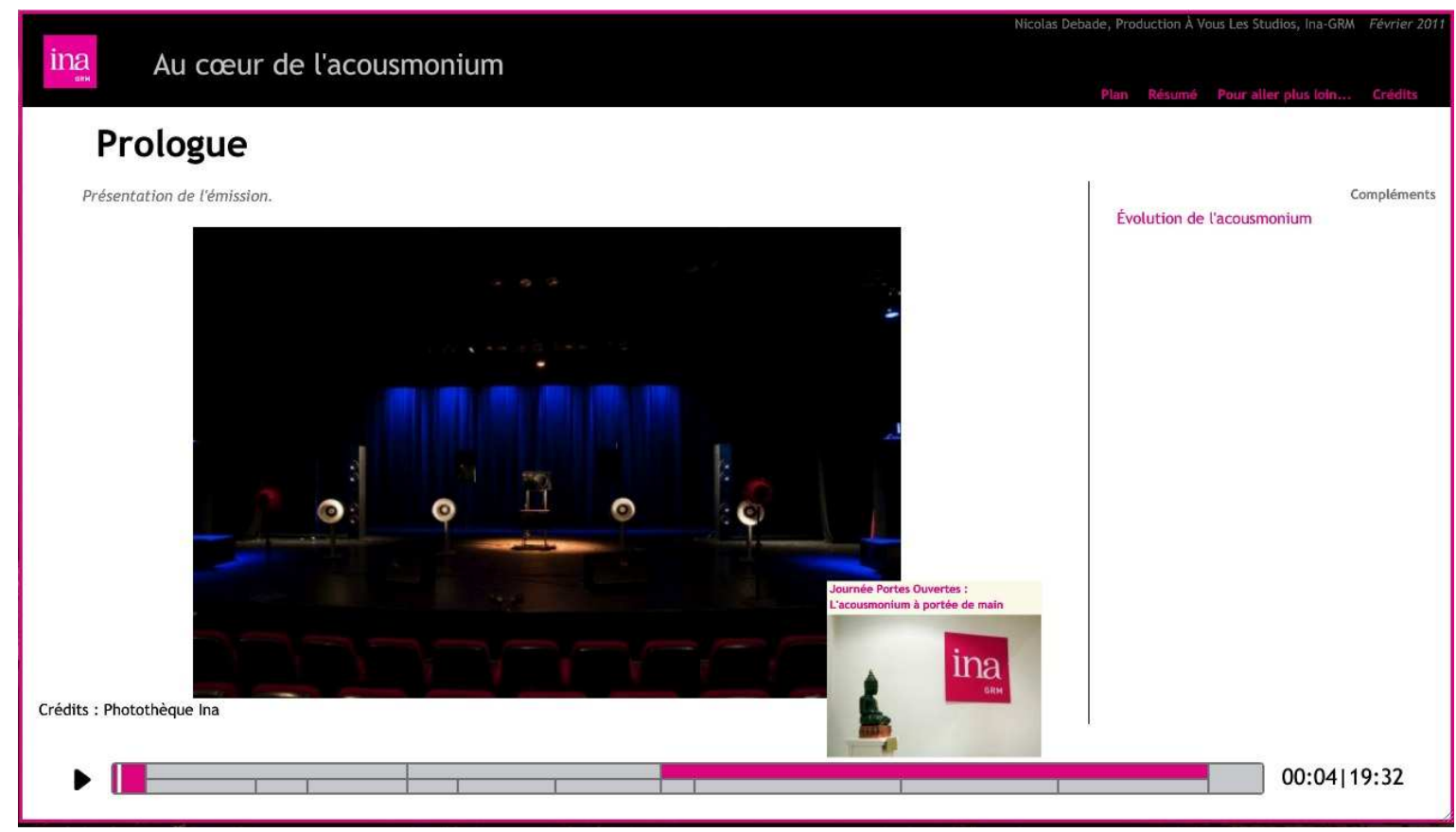

Éléments d'analyse

Le modèle documentaire Webradio doit donc favoriser le rapprochement de médias de nature différente et leur permettre de faire sens ensemble : on parle d'intersémiotisation. L'universalité du numérique lui permet de mêler indistinctement, au sein du code binaire (niveau 1), les diverses formes sémiotiques à mobiliser (niveau 3). L'art du concepteur du modèle est alors de prévoir, au niveau techno-applicatif (niveau 2), des modalités fonctionnelles qui permettent une manipulation sensée par le lecteur. Quelques exemples basiques : un texte illustratif synchronisé à l'audio maître doit être court (comme une accroche, un titre) ; un texte complémentaire peut en revanche être long ; la convocation d'un enrichissement temporel, audio ou vidéo, doit arrêter le flux maître ; le nombre d'images doit rester raisonnable par rapport au temps pendant lequel elles sont présentées.

Afin de composer avec ces contraintes, une idée structurante du modèle est que les enrichissements s'appuient sur la structure interne du flux audio original. Le document multimédia complexe et hétérogène s'adosse alors sur une linéarité, simple et solide, celle d'un document radiophonique produit pour l'écoute pure (et non la consultation Web). Au niveau de l'interface, cela se matérialise par une succession de rectangles oblongs qui symbolisent les sections et les sous-sections du flux. Lorsque la souris survole l'un de ces rectangles apparaît une illustration de la section avec son titre. Le modèle sert ici de guide pour l'écriture multimédia : des connaissances métier y sont inscrites et encadrent la production. Dans notre exemple, une image et un titre doivent faciliter la navigation du lecteur qui fenillette et lui permettre d'anticiper le contenu. 
La publication de type Webradio est encadrée par un modèle d'écriture multimédia, ellemême déterminée par des contraintes propres au numérique. Le niveau 1 renvoie à la discrétisation et à la manipulabilité des contenus. Au niveau 2, le modèle pose des règles d'écriture qui favorisent le dialogue entre la continuité du flux audio, la segmentation en fragments discrets et l'intersémiotisation.

Dans le cas de la Webradio, les idées qui ont présidé à la conception du dispositif héritent d'une réflexion sémiotique (au niveau 3) : en particulier sur la nature de type flux linéaire de l'audio dans la radio et la nature de type stock navigable de l'audio sur le Web (SaintMartin, 2007). Mais la Webradio hérite également d'une réflexion applicative (au niveau 2), notamment pour penser les limites à imposer à l'écriture, afin de la garder dans un cadre signifiant a priori qui lui a été dévolu. Ainsi une Webradio du GRM témoigne à la fois de la prise de conscience, aiguë, que le numérique ouvre des possibles nouveaux, et de l'injonction, forte, qu'il faut les limiter pour en maîtriser le sens. Là où le support permet une multitude de segmentations, une multitude d'enrichissements, la réalisation n'en retient que quelques modalités : une discrétisation modérée, un enrichissement raisonnable.

\section{CONCLUSION}

Une approche conceptuelle fondée sur les possibles du numérique permet de dégager les spécificités et les enjeux de l'écriture multimédia. L'analyse de pratiques professionnelles d'écriture multimédia illustre l'apport d'une telle approche conceptuelle quand il s'agit de lire la dynamique des usages qui se tissent autour de ces nouveaux dispositifs d'écriture.

Pour explorer les possibles de l'écriture multimédia, une autre piste à exploiter serait celle des créations numériques, littéraires (Balpe, 2006 ; Bouchardon, 2009) et artistiques (Couchot et Hillaire, 2003). Il existe en effet depuis plusieurs décennies des ouvres numériques conçues pour être lues et agies avec un ordinateur. Ces créations numériques, en tant que créations expérimentales, proposent des cas-limites. Nous constatons dès lors qu'elles peuvent jouer un rôle de révélateur de tensions entre les différents niveaux que nous avons distingués : elles provoquent et rendent observables ces tensions (Bouchardon, 2011). Notre approche conceptuelle permet dès lors d'éclairer ces pratiques de création et la façon dont les auteurs et les artistes numériques inventent et expérimentent des possibles. 


\section{Remerciements}

Nous remercions vivement Dominique Saint-Martin et Ludovic Gaillard, de l'Ina, pour leur aide indispensable et la qualité de leur accueil.

\section{REFERENCES BIBLIOGRAPHIQUES}

Bachimont, B. (2000), «L'intelligence artificielle comme écriture dynamique : de la raison graphique à la raison computationnelle ». In Petitot, J. \& Fabbri, P. (Éd.), Au nom du sens (pp. 290319). Paris : Grasset.

Bachimont, B. (2007), Ingénierie des connaissances et des contenus: Le numérique entre ontologies et documents. Paris: Hermès.

Balpe, J.-P. (2006), «Après le livre... ». In Balpe, J.-P. \& De Barros, M. (Éd.), L'art a-t-il besoin du numérique? Colloque de Cerisy. Paris : Hermès-Lavoisier.

Bouchardon, S. (2009). Littérature numérique : le récit interactif. Paris : Hermès Science.

Bouchardon, S. (2011). «Digital Literature and the Digital », Journal of Writing in Creative Practice (JWCP), vol. 4.1. Londres : Intellect Books.

Christin, A-M., (2001), Histoire de l'écriture, de l'idéogramme au multimédia. Paris: Flammarion.

Couchot, E., Hillaire, N. (2003), L’Art numérique. Paris : Flammarion.

Crozat, S., (2007), Scenari. La chaîne éditoriale libre: structurer et publier textes, images \& son. Paris : Eyrolles.

Gaillard, L., Crozat, S., (2011), Perspectives pour une (re)éditorialisation collaborative de contenus audiovisuels. In Documentaliste - sciences de l'information, dossier "Vidéo en ligne", 47, 4.

Goody, J., (1979), La raison graphique : la domestication de la pensée sauvage. Paris: Éditions de Minuit.

Saint-Martin, D., Crozat S., (2007), «Écouter, approfondir : Perspectives d'usage d'une radio interactive », Distances et savoirs 5, no. 2: 257-273.

Saint-Martin, D., Gaillard, L., Chauve, G., Carpentier, V., Poinsart, S. (2011), Expression de besoins, projet ANR C2M.

[http://scenari.utc.fr/c2m/DOCS/L4a/html/]

[http://scenari.utc.fr/c2m/DOCS/LAa/pdf/] 
